\title{
Modelling of Indian Stock Prices using Nonhomogeneous Poisson Processes with Time Trends
}

\author{
Rupal Shah and K. Muralidharan \\ Department of Statistics, Faculty of Science, \\ The Maharaja Sayajirao University of Baroda, Vadodara - 390 002, India.
}

\begin{abstract}
The financial sector in India has undergone radical reforms, particularly in the stock market segment, since early 1990s. Testing duration in stock markets concerns the ability to predict the turning points of bull and bear cycles. This article study some point process models to fit the data from Indian stock market cycles. We have considered the BSE 30 (SENSEX) data from January, 1991 to August, 2012 for bull and bear markets. The duration dependence of stock market cycles can help to pinpoint the peaks and troughs in these cycles. Upon carrying out various statistical procedures and goodness of fit tests, we found that the Nonhomogeneous Poisson Process models like Power Law Process, Modulated Power Law Process, Log-linear process and other models are some of the possible alternative models to describe the data. We provide estimates, confidence interval estimates and tests of hypothesis for the parameters involved in a particular model.
\end{abstract}

Key Words: Power Law Process, Log linear process.

\section{Introduction}

The study of stock market data efficiency has been the objective of many researches since the last few decades. The financial integration has been expected to stimulate financial efficiency and economic growth, and reduce the negative effects of shocks through more diversification and capital mobility. The Indian capital market is no longer isolated from the global economic environment.Recently India has witnessed greater volatility in its stock market. Some of which had their origin in global events like US sub-prime crisis. Inflation rates, global energy prices, exchange rate fluctuations, etc. are witnessing constant changes in the recent years. These are affecting the volatility and thus, the efficiency of the capital market. In this regard, the study of capital market efficiency in India is very significant and meaningful.

Mishra, Das and Pradhan [1] studied empirically the efficiency of Indian stock market in the context of recent global financial crisis, assuming the stock prices follow a Random Walk. Mishra [2] examined the efficiency of Indian stock market, particularly that of Stock Exchange, Mumbai (BSE) over a period of 18 years spanning from Jan, 1991 to Jan, 2009 using Random Walk (RW) and Generalised Autoregressive Conditional Heteroscedasticity $(\mathrm{GARCH})$ models. Both these studies provided the evidence of weak form market inefficiency in India.

Capital market volatility is often described as the rate and magnitude of changes in prices and in finance often referred to as risk.To a certain extent, market volatility is unavoidable, even desirable, as the stock price fluctuation indicates changing values across economic activities and it facilitates better resource allocation.But frequent and wide capital market variations cause uncertainty about the value of an asset and affect the confidence of the investor.The stock exchange prices play an important role as indicators, reflecting the performance of the country's economic state of health. It is exposed to a high degree of volatility; prices fluctuate within minutes and are determined by the demand and supply of stocks at a given time. Testing duration in stock markets concerns the ability to predict the turning points of bull and bear cycles. The Power Law process has been used in previous studies to analyze duration dependence in economic and financial cycles.Zhou and Rigdon ([3], [4]) analyzed duration dependence in U. S. business cycles analysis using the modulated power law process.

There are very few studies which address the issues of modelling the stock prices either parametrically or non-parametrically. We fill this gap by treating the time to occurrence of trough and peak of stock prices through a stochastic point process models.

We have considered the BSE 30 (SENSEX) data from January, 1991 to August, 2012 (more than 21 years span) for bull (peaks) and bear (troughs) markets. The duration dependence of stock market cycles can help to pinpoint the peaks and troughs in these cycles.

We study some point process models to fit the data from Indian stock market cycles. Upon carrying out various goodness of fit tests, we found that the Nonhomogeneous Poisson Process (NHPP) models like Power Law Process (PLP), MPLP (Modulated Power Law Process), Log-linear process (LLP) and other models are some of the possible alternative models to describe the data. 
In section 2, some point process models have been discussed. For PLP and LLP, the likelihood equation and the point estimators are suggested. Section 3 discusses about the estimation of reliability indices for PLP and LLP. In section 4, we discuss some graphical and analytical procedures to test the presence of trend and the suitability of the point process model to the given set of data. In section 5, we provide point estimates, confidence interval estimates, several other reliability indices and also carry out tests of hypothesis for testing the suitability of particular model.

\section{Nonhomogeneous poisson process models}

A non-repairable system is the one which is discarded the first time that it fails to perform satisfactorily. For such a system, the times between failures are independent and identically distributed. A repairable system is the one which, after failure to perform at least one of its required functions, can be restored to performing all of its required functions by any method, other than replacement of the entire system (Asher and Feingold [5], Calabria and Pulcini [6], Lawless [7]). The most commonly used point process models which have been applied to repairable systems are the Homogeneous Poisson Process (HPP), the Nonhomogeneous Poisson Process (NHPP), the Branching Poisson Process (BPP), the Renewal Process (RP) and the Superimposed Renewal Process (SRP) (Muralidharan [8]).

The HPP can be simply defined as a sequence of independent and identically exponentially distributed random variables $X_{i}^{\prime}$ 's.For the Renewal process, the probability of an event in a small interval depends only on the time since the previous event and not on the previous pattern of failures or the time since the process initially began. Thus, this model assumes that after the occurrence of an event, the system is always in exactly the same condition, precluding the possibility of a long term change in the system (Lawless and Thiagarajah[9]). Through goodness-of-fit tests, it can be verified whether the data fits the Renewal process model.

The Nonhomogeneous Poisson Process (NHPP) is another model that has been used to model the occurrence of events in time. For NHPP, the probability of an event in a small interval is some function of time since the initial start-up of the system, therefore, an event and the subsequent restarting of the system has no effect on the system performance. If the probability of an event occurring in a small interval is constant across time, then the process is a homogeneous Poisson process where the times between events are independent and identically distributed exponential random variables. Thus, a renewal process can be described as a good-as-new or same-as-new model, whereas the NHPP is described as a bad-as-old or same-as-old model. Therefore, a renewal process can model duration dependence but not any long term effects, such as the tendency of intervals to get longer or shorter, whereas the NHPP can model long term effects, but not duration dependence (Cox [10], [11]). An NHPP is often used to model the failures of repairable systems if they undergo minimal repairs and if there is a trend in the TBF. If the TBF are monotonically increasing (decreasing), then this indicates that failures are becoming less (more) frequent and that the system is improving (deteriorating) as the operating time increases (Cox and Lewis [12]).

In our paper, the discussions and the results are based on the analysis of the times between successive events of the SENSEX, where event is peak or trough, which we denote as time between successive failures (TBFs). When a trend is noticed in the data set, we describe the trend using the NHPP models like, Power Law process and Log-linear process, which assumes that the TBFs vary as a function of time. We provide estimates, confidence interval estimates and tests of hypothesis for the parameters involved in a particular model.

\subsection{The Power Law Process}

The PLP has proved to be a useful model for analyzing systems which are deteriorating or improving with time (Rigdon and Basu[13]). For PLP, the probability of an event in a small interval depends only on the time since the previous event and not on the previous pattern of failures or the time since the process initially began.Thus, this model assumes that after the occurrence of an event, the system is always in exactly the same condition.

The intensity function of PLP is given by:

$$
u(t)=\frac{\beta}{\theta}\left(\frac{t}{\theta}\right)^{\beta-1}
$$

where parameters $\theta$ and $\beta$ are scale and shape parameters, respectively. If $1<\beta<2$, the intensity function is concave and increases more slowly with operating time, and if $\beta>2$, the intensity function is convex. Crow ([14], [15]) obtained maximum likelihood estimates of the parameter $\theta$ and $\beta$ and discussed methods of performing hypothesis tests and constructing confidence limits for these parameters. Baker [16] suggested some tests for checking whether the process is PLP.Rigdon and Basu[17] discussed the procedure of estimating the intensity function of PLP at current time.

Let $0<t_{1}<t_{2}<\ldots<t_{n}$ denote the first $\mathrm{n}$ ordered system failure times from a time-truncated power law process 
with the intensity function as given above. The joint density or the likelihood function of $t_{1}, t_{2}, \ldots, t_{n}$, for an NHPP is given by:

$$
L\left(t_{1}, t_{2}, \ldots, t_{n}\right)=\prod_{i=1}^{n} u\left(t_{i}\right) \exp \left\{-\Lambda\left(t_{n}\right)\right\},
$$

Where the cumulative intensity function is:

$$
\Lambda(t)=\int_{0}^{t} u(x) d x(3)
$$

and $t_{0}=0$.

Thus, for the PLP, the likelihood function of $t_{1}, t_{2}, \ldots, t_{n}$ will be:

The log likelihood function will be :

$$
L=(\beta / \theta)^{n} \exp \left[-\left(t_{n} / \theta\right)^{\beta}\right] \prod_{i=1}^{n}\left(t_{i} / \theta\right)^{\beta-1}
$$

$$
\ln L=\ell=n \ln (\beta / \theta)-\left(t_{n} / \theta\right)^{\beta}+(\beta-1) \sum_{i=1}^{n} \ln \left(t_{i} / \theta\right)
$$

This gives the ML estimates of $\theta$ and $\beta$ as:

and

$$
\hat{\theta}=\frac{t_{n}}{n^{1 / \widehat{\beta}}}
$$

$$
\hat{\beta}=\frac{n}{\sum_{i=1}^{n-1} \ln \left(t_{n} / t_{i}\right)}
$$

(7)can be solved first analytically to obtain the estimate of $\beta$ and then using this estimate in (6), the estimate of $\theta$ can be obtained.

\subsection{The Log-Linear Process (Cox Process)}

If the power law model is rejected by a goodness-of-fit test, other NHPP models can be fitted and tested for goodness-of-fit. One such model was proposed by Cox and Lewis [12] with intensity function:

$$
u(t)=\exp (a+b t),-\infty<a, b<\infty
$$

where $a$ is the scale parameter and $b$ is the shape parameter.

Note that $u(t)$ is strictly decreasing for $b<0$, a constant for $b=0$, and strictly increasing for $b>0$. Thus this model has a monotonic trend in the failure data indicating system reliability improvement when $b<0$ and deterioration when $b>0$. The LLP reduces to HPP when $b=0$. The most important characteristic of the LLP model is that its failure intensity function is greater than 0 at $t=0$ and is convex for any value of $b$. This characteristic makes the LLP model suitable to describe failure process of repairable system with extremely fast increasing failure rate.

The likelihood function for LLP is given by:

And the log likelihood of the process is given by:

$$
L=\exp (a n) \exp \left[\left(b \sum_{i=1}^{n} t_{i}\right)-\frac{\exp (a)}{b}\left(\exp \left(b t_{n}-1\right)\right)\right]
$$

$$
\log L=\ell=n a+b \sum_{i=1}^{n} t_{i}-\frac{\exp (a)}{b}\left(\exp \left(b t_{n}-1\right)\right)
$$

Differentiating the $\log$ likelihood function with respect to parameters $a$ and $b$ gives the following likelihood equations:

$$
\begin{aligned}
& \frac{1}{\hat{b}}=\frac{t_{n} \exp \left(b t_{n}\right)}{\left(\exp \left(b t_{n}\right)-1\right)}-\frac{\sum_{i=1}^{n} t_{i}}{n} \\
& \hat{a}=\ln \left(\frac{n \hat{b}}{\exp \left(\hat{b} t_{n}-1\right)}\right)
\end{aligned}
$$

Solve (11) for $b$ using an iterative method and substitute this estimate in (12) to obtain the estimate of $a$.Wang and $\mathrm{Xu}[18]$ discussed the procedures of obtaining point and interval estimates of parameters and other reliability indices for log-linear process model for NC machine tools. Muralidharan and Shah [19] derived the conditional test for trend in process.

\section{Estimation procedure}

Using the log likelihood function for PLP and LLP, the second order derivatives and partial derivatives of parameters can be obtained as, respectively:

$$
\Delta_{11}=-\frac{\partial^{2} \ell}{\partial \theta^{2}}, \Delta_{12}=\Delta_{21}=-\frac{\partial^{2} \ell}{\partial \theta \partial \beta}, \quad \Delta_{22}=-\frac{\partial^{2} \ell}{\partial \beta^{2}}
$$




$$
\Delta_{11}=-\frac{\partial^{2} \ell}{\partial a^{2}}, \Delta_{12}=\Delta_{21}=-\frac{\partial^{2} \ell}{\partial a \partial b}, \quad \Delta_{22}=-\frac{\partial^{2} \ell}{\partial b^{2}}
$$

Since the pivotal quantities of the parameters are not available, a common approach for obtaining asymptotic confidence regions for model parameters is to use the asymptotic theory of maximum likelihood estimation. In a general framework, let $\boldsymbol{\gamma}$ be the model parameter and $\hat{\gamma}$ be its maximum likelihood estimator.When observed data are independent and identically distributed, it is well known that the asymptotic distribution of $\hat{\gamma}$ is given by:

$$
I_{n}^{1 / 2}[\hat{\gamma}-\gamma] \stackrel{d}{\rightarrow} N(0, I)
$$

where $I_{n}$ is the local Fisher's Information matrix. This information matrix can be used to propose approximate Confidence Interval for the parameters. The inverse of the local Fisher Information matrix obtained as follows, gives the estimate of the covariance matrix of MLE, for PLP and LLP respectively, as:

and

$$
I_{n}^{-1}=\left[\begin{array}{cc}
\operatorname{var}(\hat{\theta}) & \operatorname{cov}(\hat{\theta}, \hat{\beta}) \\
\operatorname{cov}(\hat{\theta}, \hat{\beta}) & \operatorname{var}(\hat{\beta})
\end{array}\right]=\left[\begin{array}{cc}
\Delta_{11} & \Delta_{12} \\
\Delta_{21} & \Delta_{22}
\end{array}\right]_{\theta=\hat{\theta}, \beta=\widehat{\beta}}^{-1}
$$

$$
I_{n}^{-1}=\left[\begin{array}{cc}
\operatorname{var}(\hat{a}) & \operatorname{cov}(\hat{a}, \hat{b}) \\
\operatorname{cov}(\hat{a}, \hat{b}) & \operatorname{var}(\hat{b})
\end{array}\right]=\left[\begin{array}{cc}
\Delta_{11} & \Delta_{12} \\
\Delta_{21} & \Delta_{22}
\end{array}\right]_{a=\hat{a}, b=\hat{b}}^{-1}
$$

As the MLEs are asymptotically normally distributed, we can use the asymptotic lognormal distribution for the MLEs and obtain the approximate $100(1-\alpha) \%$ confidence interval for parameter of interest, say $\gamma$, as

$$
\text { C.I. for } \gamma=\hat{\gamma} \exp \left( \pm z_{\alpha / 2} \sqrt{\operatorname{var}(\hat{\gamma})} / \hat{\gamma}\right)
$$

where $\hat{\gamma}$ is the estimated parameter of PLP or LLP, and $z_{\alpha / 2}$ is the upper $\alpha / 2$ percentile point of a standard normal distribution.The other reliability indices of interest for both PLP and LLP are: the estimate of failure intensity function $(\hat{u}(t))$, the estimate of mean time between failures $(\widehat{m}(t)=1 / \hat{u}(t))$, the estimate of cumulative failure intensity function $\left(\hat{u}_{c}(t)=\widehat{\Lambda}(t) / t=\hat{E}[N(t)] / t\right)$, the estimate of cumulative MTBF $\left(\widehat{m}_{c}(t)=\mathrm{t} / \widehat{\Lambda}(t)=t / \hat{E}[N(t)]\right)$, the estimate of reliability at a given time ' $t$ ' $\left(\hat{R}(t)=e^{-\widehat{\Lambda}(t)}\right)$, the estimate of warranty time for a given reliability $(\hat{t}(R))$, which can be obtained from equation of $R(t)$ and the estimate of number of failures up to given time ' $t$ ' $\widehat{N}(t)=\widehat{\Lambda}(t)$.

To find the confidence interval with confidence coefficient $(1-\alpha)$, the following procedure may be used:

Let $g(t)$ be the reliability index whose confidence bounds are to be computed and suppose the NHPP under consideration has two model parameters, say, $\gamma$ and $\delta$. Then compute:

$$
\operatorname{var}(\hat{g}(t))=\left(\frac{\partial \hat{g}(t)}{\partial \gamma}\right)^{2} \operatorname{var}(\hat{\gamma})+2\left(\frac{\partial \hat{g}(t)}{\partial \gamma}\right)\left(\frac{\partial \hat{g}(t)}{\partial \delta}\right) \operatorname{cov}(\hat{\gamma}, \hat{\delta})+\left(\frac{\partial \hat{g}(t)}{\partial \delta}\right)^{2} \operatorname{var}(\hat{\delta})
$$

Then the approximate $100(1-\alpha) \%$ confidence interval for $g(t)$ is given by:

$$
\text { C.I.for } g(t)=\hat{g}(t) \exp \left( \pm z_{\alpha / 2} \frac{\sqrt{\operatorname{var}(\hat{g}(t))}}{\hat{g}(t)}\right)
$$

Also the two information criteria can be used to choose the best model, namely, Akaike Information Criterion (AIC) and Bayesian Information Criterion (BIC) defined by:

$$
A I C=-2 \max \ell+2 m, B I C=-2 \max \ell+m \ln n
$$

Where, $m$ is the number of estimated parameters, $n$ is the number of observations and max $\ell$ is the maximized log-likelihood.

\section{Trend testing}

It is essential to check the existence of trend or time dependency in the Times between failures (TBFs) before selecting the suitable model for the failure intensity function. Graphical methods and analytical methods are two widely used methods of testing time-trend for the system. When a trend is noticed in the data set, one possibility to describe the trend is to use the NHPP models like PLP, LLP etc., which assumes that the TBFs vary as a function of time.

\subsection{Graphical Procedures for Analysis of Trend}

Analysis of reliability of available data is usually based on the assumption that the times between events (failures) are independent and identically distributed in the time domain. So we can consider tests for trends to check whether the assumption of i.i.d. for the failure times is satisfied or not. For which, cumulative 
number of failures can be plotted against cumulative time between successive failures. The convexity of the curve can be interpreted as the presence of trend in the dataNelson [20].

Specifically, if we are interested in checking the trend in the process, we can use the Duane plot, which is the test for checking whether the process is PLP (Duane [21]). Duane Plot can be based on the plot of $\ln (t)$ against $\ln (N(t))$, where $N(t)$ is the number of failures up to time $t$. The linearity of the plot indicates that the PLP can be a suitable model to describe the failure times.

The other alternative of Duane plot is the total time on test (TTT) plot (Klefjoand Kumar [22]). Suppose $n$ units are put on test and $0<x_{(I)}<x_{(2)}<\ldots<x_{(n)}<\infty$ be the failure times of these units. If $x_{0}=0$, then the total time on test at time $t$ is defined as:

$$
X(t)=\sum_{j=1}^{i-1}(n-j+1)\left(x_{(j)}-x_{(j-1)}\right)+(n-i+1)\left(t-x_{(i)}\right)
$$

Let $T_{i}=X\left(t_{i}\right), i=1,2, \ldots, n$ be the values of $X(t)$ at the failure times and $V_{i}=T_{i} / T_{n}$, then the graph of $(i / n)$ versus $V_{i}, i=1,2, \ldots, n$ is called the TTT plot. TTT plot can be used to study the presence of trend.

\subsection{Analytical Methods (Goodness of Fit Tests)}

4.2.1The times between failures can be analyzed for the presence of trend by using the test suggested in MILHDBK-189[23] by calculating the test statistic:

$$
U=2 \sum_{i=1}^{n-1} \ln \left(t_{n} / t_{i}\right)
$$

when the data is failure-truncated at the $n$th failure at time $t_{n}$. Under the null hypothesis of a HPP, the test statistic $U$ is chi-squared distributed with $2(n-1)$ d.f. The null hypothesis is rejected if the value of test statistic $U<$ the critical value, and thereby it can be concluded that the process is NHPP.

4.2.2 The Cramer-von-Mises (CVM) test statistic suggested by Crow [14] to test the null hypothesis that the failure times follow PLP versus the alternative that the failure times do not follow PLP is given as

$$
C_{M}^{2}=\frac{1}{12 M}+\sum_{j=1}^{M}\left\{Z_{j}^{\widehat{\beta}}-(2 j-1) / 2 M\right\}^{2}
$$

Where $M=n-l$ and $Z_{j}=\frac{t_{j}}{t}$, where $t$ is a pre-assigned time upto which the system is observed.

If the test statistic is greater than the selected critical value, then the hypothesis that the failure times follow a PLP is rejected at the selected level of significance.

4.2.3Let $t_{i}$ be the cumulative failure times. Then the Laplace Test Statistics for testing $\mathrm{H}_{0}$ : The process is HPP vs. $\mathrm{H}_{1}$ : The process is NHPP is defined as

$$
\mathrm{U}=\frac{\frac{\sum_{\mathrm{i}=1}^{\mathrm{n}-1} t_{i}}{\mathrm{n}-1}-\frac{\mathrm{t}_{\mathrm{n}}}{2}}{\mathrm{t}_{\mathrm{n}} \sqrt{\frac{1}{12(\mathrm{n}-1)}}} \sim \mathrm{N}(0,1)
$$

If the value of the test statistic $U>$ the critical value, the null hypothesis is rejected.

4.2.4Let $x_{i}=t_{i}-t_{i-1}, i=1,2, \ldots, n$ be the inter arrival times. Then the Lewis-Robinson test Statistics for testing $\mathrm{H}_{0}$ : The process is renewal process Vs. $\mathrm{H}_{1}$ : The process hasmonotonic trends is defined as

$$
U_{L R}=\frac{U}{\widehat{C . v} \cdot(x)} \sim N(0,1)
$$

Where $\mathrm{U}=$ Laplace test statistic, $\widehat{c . \widehat{v}} \cdot(x)=$ estimated coefficient of variation of $x_{i}$. If the value of test statistic $>$ critical value, $\mathrm{H}_{0}$ is rejected.

4.2.5A test based on quadratic form proposed by Janiet. al. [24] for testing $\mathrm{H}_{0}$ : The process is HPP vs. $\mathrm{H}_{1}$ : The process is NHPP has the form

$$
Q=2 n(n+1) \sum_{i=1}^{n-1} \frac{t_{i}}{t_{n}}\left(\frac{t_{i}}{t_{n}}-\frac{t_{i+1}}{t_{n}}\right)+\left(n^{2}-1\right)
$$

Under $\mathrm{H}_{0}$, the mean and variance of $Q$ are $E(Q)=n-1$ and $(Q)=\frac{4 n^{2}(n-1)}{(n+2)(n+3)}$. If the standardized value of $Q>$ critical value suggested by the authors in their article, then $\mathrm{H}_{0}$ is rejected. 


\section{Data analysis}

We consider the BSE 30 (SENSEX) data from January, 1991 to August, 2012 (more than 21 years span) for bull (peaks) and bear (troughs) markets. We are considering the monthly high for each month as the point of our interest and we consider the failure times as the time intervals (in months) after which the peak or trough occurs. Moreover, these time points are observed according to the change (up or down) of 500 points, 1000 points and 1500 points. Thus, we have considered 6 datasets, namely, 500 points up, 500 points down, 1000 points up, 1000 points down, 1500 points up and 1500 points down and for each of these datasets we get respectively, 58, 37, 31, 19, 21 and 10 observations. In each dataset, we have noted down cumulative number of months, starting from January 1991 to August, 2012 after which there was increase or decrease of 500 points, 1000 points and 1500 points in sensex, which are considered here as event times.

\section{A: 500 points UP data $(n=58)$}

Cumulative number of months:

$6,12,13,14,20,32,34,36,41,61,63,72,78,87,96,100,102,108,109,133,150,152,153,155,165,167$, $173,174,176,179,180,181,182,183,184,187,188,189,190,192,195,198,200,201,204,219,220,221$, $224,228,230,234,236,237,242,249,253,259$.
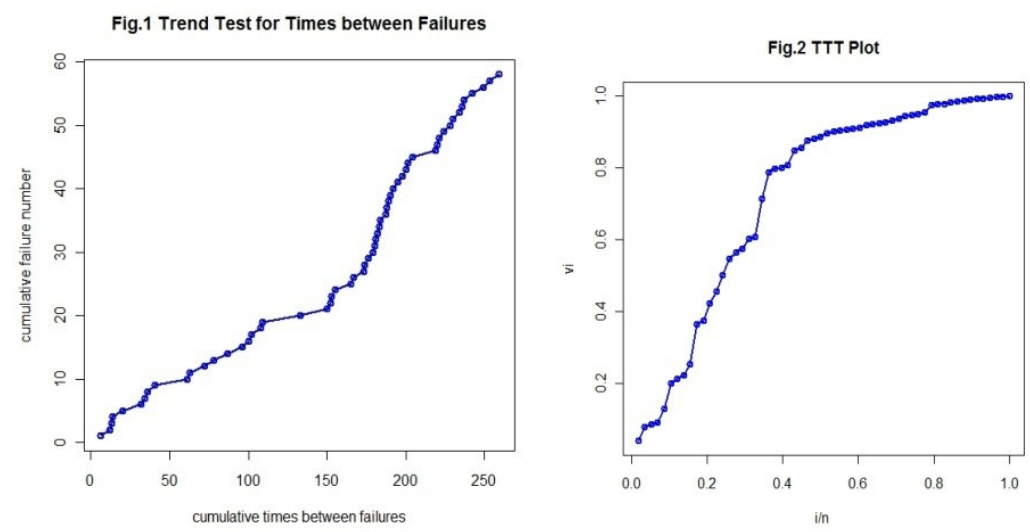

Convexity of the curve in Fig. 1 means presence of trend in the data. The TTT plot in Fig. 2 indicates that the data initially shows increasing failure rate, then there is an improvement in the system and then after again the failure rate is increasing. That means the trend is present in the data. Linearity of Duane plot indicates that the data follows PLP, so here from Fig. 3, we can say that the process is PLP.

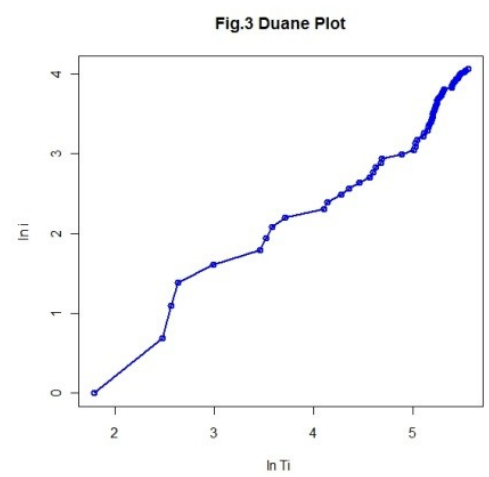

Table 1 shows the results of goodness of fit tests discussed is section 4.2, from which it can be concluded that the data can be modelled using an NHPP. Table 2 and 3 consists of point and interval estimates of model parameters and reliability indices for PLP and LLP respectively. In Table 4, the values of AIC and BIC computed using (18) are shown for the purpose of deciding the suitability of PLP or LLP model for the data, which shows that LLP is a more suitable model for describing the 500 points up data.

Table 1 Goodness of fit Tests

\begin{tabular}{|l|c|c|c|}
\hline & Test statistic value & Critical Value & Conclusion \\
\hline MIL-HDBK-189 Test & 90.91565 & 139.9208 & Process is NHPP \\
\hline CVM Test & 0.2115203 & 0.22 & Process is PLP \\
\hline Laplace Test & 1.995651 & 1.96 & Process is NHPP \\
\hline Lewis-Robinson Trend Test & 1.844132 & 1.96 & Process is NHPP \\
\hline Standardized Q & 0.8354 & -1.3368 & Process is NHPP \\
\hline
\end{tabular}


Table 2 Point and Interval estimates of reliability indices using PLP

\begin{tabular}{|c|c|c|}
\hline & Point Estimate & Interval Estimate \\
\hline $\boldsymbol{\theta}$ & 10.74492 & $(4.622508,24.97633)$ \\
\hline $\boldsymbol{\beta}$ & 1.275908 & $(0.0963908,1.650402)$ \\
\hline $\mathbf{u}(\mathbf{t})$ & 0.2857245 & $(1.202281,10.18823)$ \\
\hline $\mathbf{m}(\mathbf{t})$ & 3.499874 & $(0.1731243,0.2896667)$ \\
\hline $\mathbf{u}_{\mathbf{c}}(\mathbf{t})$ & 0.2239382 & $(3.452244,5.776198)$ \\
\hline $\mathbf{m}_{\mathbf{c}}(\mathbf{t})$ & 4.465517 & $(44.83918,75.02367)$ \\
\hline $\mathbf{N}(\mathbf{t})$ & 58 & $\left(2.129331 \times 10^{-32}, 1.966061 \times 10^{-19}\right)$ \\
\hline $\mathbf{R}(\mathbf{t}=\mathbf{2 5 9})$ & $6.470235 \times 10^{-26}$ & $(0.01051144,0.0242085)$ \\
\hline $\mathbf{t}(\mathbf{R}=\mathbf{0 . 9})$ & 0.015952 & \\
\hline
\end{tabular}

Table 3 Point and Interval estimates of reliability indices using LLP

\begin{tabular}{|c|c|c|}
\hline & Point Estimate & Interval Estimate \\
\hline $\boldsymbol{a}$ & -2.004379 & $(-2.789089,-1.440446)$ \\
\hline $\boldsymbol{b}$ & 0.0039377 & $(0.001479899,0.01048963)$ \\
\hline $\mathbf{u}(\mathbf{t})$ & 0.3738433 & $(1.677687,4.264911)$ \\
\hline $\mathbf{m}(\mathbf{t})$ & 2.674918 & $(0.1808834,0.303505)$ \\
\hline $\mathbf{u}_{\mathbf{c}}(\mathbf{t})$ & 0.2343054 & $(3.294839,5.528423)$ \\
\hline $\mathbf{m}_{\mathbf{c}}(\mathbf{t})$ & 4.267934 & $(46.8488,78.6078)$ \\
\hline $\mathbf{N}(\mathbf{t})$ & 60.6851 & $\left(6.680199 \times 10^{-34}, 2.916116 \times 10^{-20}\right)$ \\
\hline $\mathbf{R}(\mathbf{t}=\mathbf{2 5 9})$ & $4.413642 \times 10^{-27}$ & $(0.402482,1.514447)$ \\
\hline $\mathbf{t}(\mathbf{R}=\mathbf{0 . 9})$ & 0.780729 & \\
\hline
\end{tabular}

Table 4 Comparison on the basis of AIC and BIC

\begin{tabular}{|c|c|c|c|}
\hline & AIC & BIC & Conclusion \\
\hline PLP & 290.4007 & 294.5216 & LLP is better than PLP for \\
this data.
\end{tabular}

\section{B: 500 points DOWN data $(n=37)$}

Cumulative number of months:

$16,17,22,27,47,49,67,71,80,85,89,91,111,112,115,117,123,129,139,160,161,194,209,210,212$, $213,214,215,217,229,232,241,245,247,248,251,256$.

Convexity of the curve in Fig. 4 means presence of trend in the data. The TTT plot in Fig. 5 indicates that the data initially shows increasing failure rate, then there is an improvement in the system and then after again the failure rate is increasing. That means the trend is present in the data. Linearity of Duane plot in Fig. 6 indicates that the process is PLP.
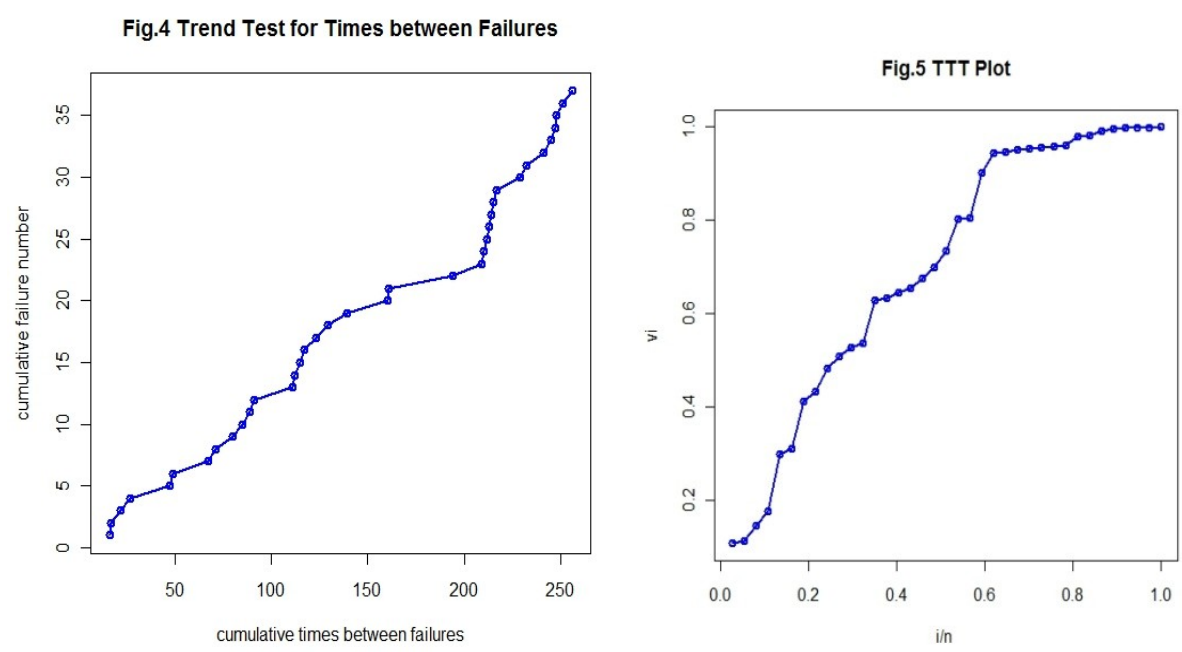

From Table 5, it can be concluded that the data can be modelled using an NHPP. Table 6 and 7 consists of point and interval estimates of model parameters and reliability indices for PLP and LLP respectively. FromTable 8, we can conclude that LLP is a more suitable model for describing the 500 points down data. 


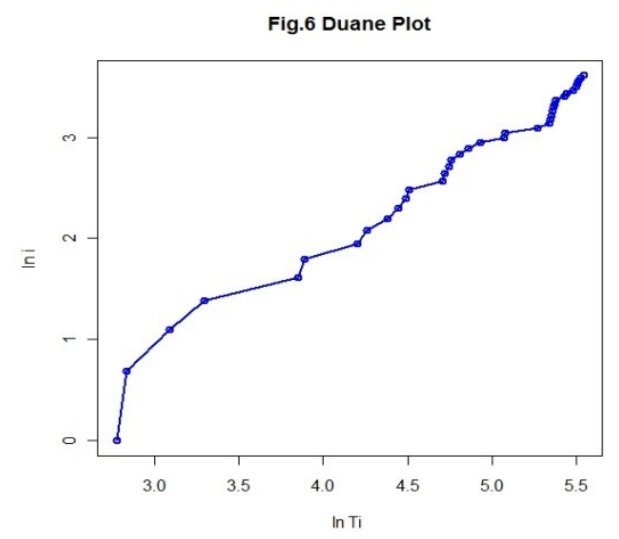

Table 5 Goodness of fit Tests

\begin{tabular}{|l|c|c|c|}
\hline & Test statistic value & Critical Value & Conclusion \\
\hline MIL-HDBK-189 Test & 57.39107 & 92.80827 & Process is NHPP \\
\hline CVM Test & 0.1099907 & 0.2184 & Process is PLP \\
\hline Laplace Test & 1.346399 & 1.96 & Process is HPP \\
\hline Lewis-Robinson Trend Test & 1.251894 & 1.96 & Process is NHPP \\
\hline Standardized Q & 0.707539 & -1.30 & Process is NHPP \\
\hline
\end{tabular}

Table 6 Point and Interval estimates of reliability indices using PLP

\begin{tabular}{|c|c|c|}
\hline & Point Estimate & Interval Estimate \\
\hline $\boldsymbol{\theta}$ & 15.56013 & $(6.100533,39.68797)$ \\
\hline $\mathbf{u}(\mathbf{t})$ & 1.289399 & $(0.9342179,1.779617)$ \\
\hline $\mathbf{m}(\mathbf{t})$ & 0.1863585 & $(0.04168006,0.8332398)$ \\
\hline $\mathbf{u}_{\mathbf{c}}(\mathbf{t})$ & 5.366002 & $(0.1047183,0.1994808)$ \\
\hline $\mathbf{m}_{\mathbf{c}}(\mathbf{t})$ & 0.1445313 & $(5.013015,9.54943)$ \\
\hline $\mathbf{N}(\mathbf{t})$ & 6.918919 & $(26.80788,51.06707)$ \\
\hline $\mathbf{R}(\mathbf{t}=\mathbf{2 5 6})$ & 37 & $(5.666986 \mathrm{e}-22,1.284861 \mathrm{e}-11)$ \\
\hline $\mathbf{t}(\mathbf{R}=\mathbf{0 . 9})$ & $8.533048 \mathrm{e}-17$ & $(0.007358066,0.01711101)$ \\
\hline
\end{tabular}

Table 7 Point and Interval estimates of reliability indices using LLP

\begin{tabular}{|c|c|c|}
\hline & Point Estimate & Interval Estimate \\
\hline $\boldsymbol{a}$ & -2.435954 & $(-3.168135,-1.703773)$ \\
\hline $\mathbf{b}(\mathbf{t})$ & 0.003639 & $(0.001069913,0.012377)$ \\
\hline $\mathbf{m}(\mathbf{t})$ & 0.2221572 & $(2.518903,8.043917)$ \\
\hline $\mathbf{u}_{\mathbf{c}}(\mathbf{t})$ & 4.501316 & $(0.1047183,0.1994808)$ \\
\hline $\mathbf{m}_{\mathbf{c}}(\mathbf{t})$ & 0.1445313 & $(5.013015,9.54943)$ \\
\hline $\mathbf{N}(\mathbf{t})$ & 6.918919 & $(26.80788,51.06708)$ \\
\hline $\mathbf{R}(\mathbf{t}=\mathbf{2 5 6})$ & 37 & $(0.5771906,2.50023)$ \\
\hline $\mathbf{t}(\mathbf{R}=\mathbf{0 . 9})$ & $8.53303 \times 10^{-17}$ & $\left(566971 \times 10^{-22}, 1.284859 \times 10^{-11}\right)$ \\
\hline
\end{tabular}

Table 8 Comparison on the basis of AIC and BIC

\begin{tabular}{|c|c|c|c|}
\hline & AIC & BIC & Conclusion \\
\hline PLP & 253.6080 & 256.8298 & $\begin{array}{c}\text { LLP is better than PLP for } \\
\text { this data. }\end{array}$ \\
\hline
\end{tabular}

\section{C: 1000 points $U P(n=31)$}

Cumulative number of months:

$12,14,35,100,105,109,151,155,166,173,176,180,182,184,188,190,193,195,198$, 200, 201, 204, 219, 220, 223, 224, 231, 236, 238, 243, 253.

Convexity of the curve in Fig. 7 means presence of trend in the data. The TTT plot in Fig. 8 indicates that the data initially shows increasing failure rate, then there is an improvement in the system and then after again the failure rate is increasing. That means the trend is present in the data. Duane plot in Fig. 9 exhibits nonlinearity, which indicates that the process is not PLP. 
From Table 9, it can be concluded that the data can be modelled using an NHPP. Table 10 and 11 consists of point and interval estimates of model parameters and reliability indices for PLP and LLP respectively. Table 12 shows that LLP is a more suitable model for describing the 1000 points up data.
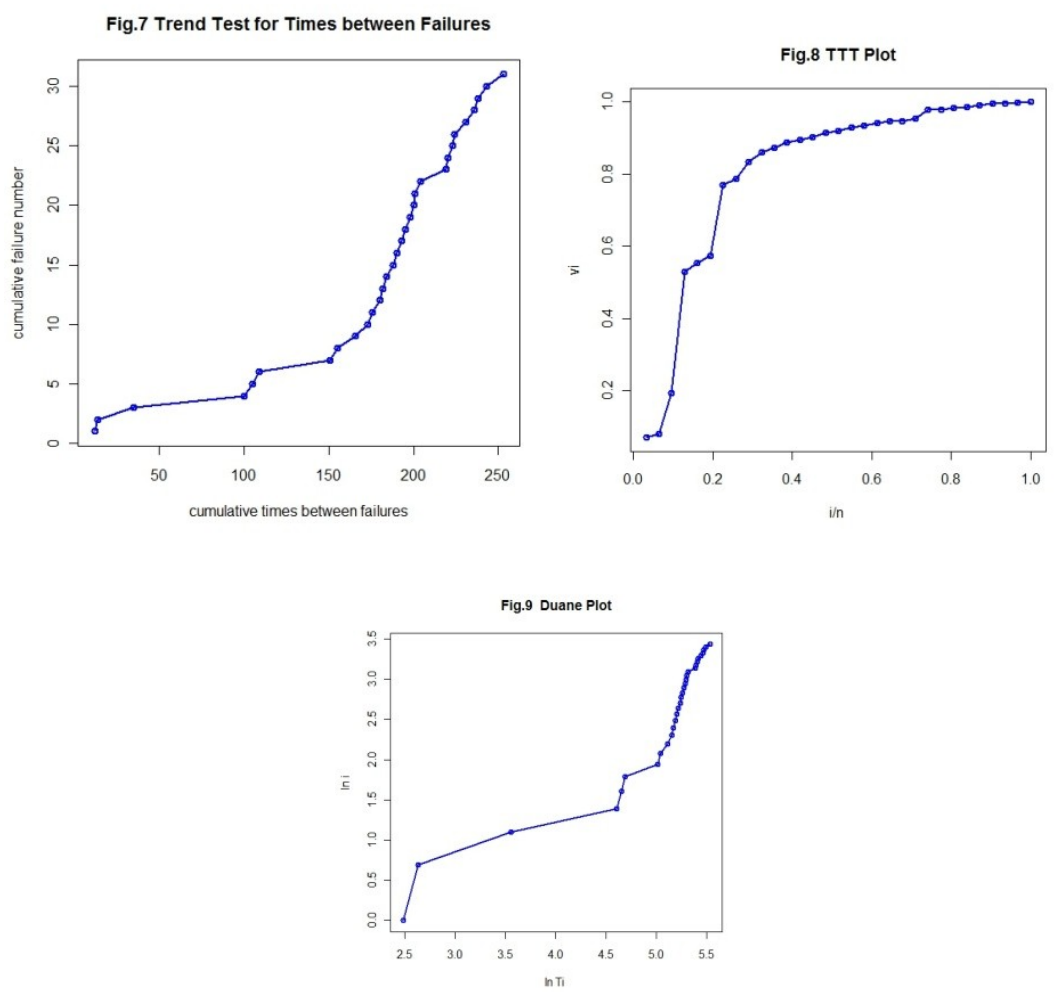

Table 9 Goodness of fit Tests

\begin{tabular}{|l|c|c|c|}
\hline & Test statistic value & Critical Value & Conclusion \\
\hline MIL-HDBK-189 Test & 33.12653 & 79.08194 & Process is NHPP \\
\hline CVM Test & 0.2283568 & 0.218 & Process is not PLP \\
\hline Laplace Test & 3.374763 & 1.96 & Process is NHPP \\
\hline Lewis-Robinson Trend Test & 2.112769 & 1.96 & Process is HPP \\
\hline Standardized Q & 5.0942093 & -1.2723 & Process is NHPP \\
\hline
\end{tabular}

Table 10 Point and Interval estimates of reliability indices using PLP

\begin{tabular}{|c|c|c|}
\hline & Point Estimate & Interval Estimate \\
\hline $\boldsymbol{\theta}$ & 40.39129 & $(20.61247,79.14903)$ \\
\hline $\mathbf{u}(\mathbf{t})$ & 1.871612 & $(0.01316233,2.661331)$ \\
\hline $\mathbf{m}(\mathbf{t})$ & 0.2293279 & $(0.2622621,72.50208)$ \\
\hline $\mathbf{u}_{\mathbf{c}}(\mathbf{t})$ & 4.360568 & $(0.08617039,0.1742305)$ \\
\hline $\mathbf{m}_{\mathbf{c}}(\mathbf{t})$ & 0.1225296 & $(5.739522,11.60491)$ \\
\hline $\mathbf{N}(\mathbf{t})$ & 8.16129 & $(21.80111,44.08033)$ \\
\hline $\mathbf{R}(\mathbf{t}=\mathbf{2 5 3})$ & 31 & $(6.273275 \mathrm{e}-19,1.889069 \mathrm{e}-09)$ \\
\hline $\mathbf{t}(\mathbf{R}=\mathbf{0 . 9})$ & $3.442477 \mathrm{e}-14$ & $(0.005558514,0.009956385)$ \\
\hline
\end{tabular}

Table 11 Point and Interval estimates of reliability indices using LLP

\begin{tabular}{|c|c|c|}
\hline & Point Estimate & Interval Estimate \\
\hline $\boldsymbol{a}$ & -3.584452 & $(-4.775969,-2.690196)$ \\
\hline $\boldsymbol{b}$ & 0.00979 & $(0.005567898,0.01727274)$ \\
\hline $\mathbf{u}(\mathbf{t})$ & 0.3317619 & $(1.718624,5.286473)$ \\
\hline $\mathbf{m}(\mathbf{t})$ & 3.01421 & $(0.08617039,0.1742305)$ \\
\hline $\mathbf{u}_{\mathbf{c}}(\mathbf{t})$ & 0.1225296 & $(5.739522,11.60491)$ \\
\hline $\mathbf{m}_{\mathbf{c}}(\mathbf{t})$ & 8.16129 & $(21.80111,44.08033)$ \\
\hline $\mathbf{N}(\mathbf{t})$ & 31 & $\left(6.273257 \times 10^{-19}, 1.889069 \times 10^{-9}\right)$ \\
\hline $\mathbf{R}(\mathbf{t}=\mathbf{2 5 3})$ & $3.442477 \times 10^{-14}$ & $(1.344483,10.33455)$ \\
\hline $\mathbf{t}(\mathbf{R}=\mathbf{0 . 9})$ & 3.72755 & \\
\hline
\end{tabular}


Table 12 Comparison on the basis of AIC and BIC

\begin{tabular}{|c|c|c|c|}
\hline & AIC & BIC & Conclusion \\
\hline PLP & 186.1748 & 189.0428 & LLP is better than PLP for \\
this data.
\end{tabular}

\section{D: 1000 points DOWN $(n=19)$}

Cumulative number of months:

27, 49, 71, 85, 91, 112, 127, 161, 185, 205, 206, 209, 210, 213, 214, 229, 241, 248, 251.

Convexity of the curve in Fig. 10 means presence of trend in the data. The TTT plot in Fig. 11 indicates that the data initially shows increasing failure rate, then there is an improvement in the system and then after again the failure rate is increasing. That means the trend is present in the data. Linearity of Duane plot in Fig. 12 indicates that the process is PLP.
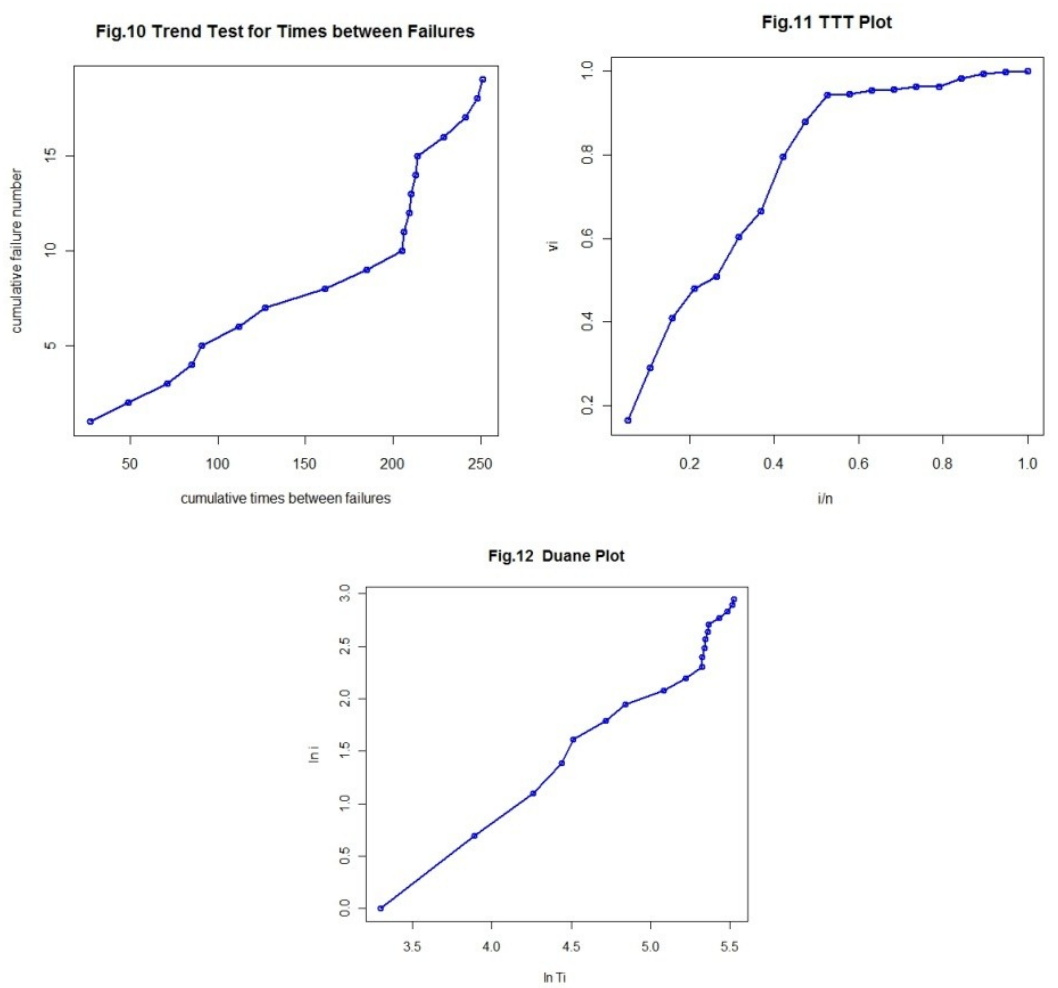

From Table 13, it can be concluded that the data can be modelled using an NHPP. Table 14 and 15 consists of point and interval estimates of model parameters and reliability indices for PLP and LLP respectively. Table 16 shows that PLP is a more suitable model for describing the 1000 points down data.

Table 13 Goodness of fit Tests

\begin{tabular}{|l|c|c|c|}
\hline & Test statistic value & Critical Value & Conclusion \\
\hline MIL-HDBK-189 Test & 21.38063 & 50.99846 & Process is NHPP \\
\hline CVM Test & 0.09271252 & 0.217 & Process is PLP \\
\hline Laplace Test & 2.029856 & 1.96 & Process is NHPP \\
\hline Lewis-Robinson Trend Test & 2.710841 & 1.96 & Process is HPP \\
\hline Standardized Q & -0.90474967 & -1.223 & Process is NHPP \\
\hline
\end{tabular}

Table 14 Point and Interval estimates of reliability indices using PLP

\begin{tabular}{|c|c|c|}
\hline & Point Estimate & Interval Estimate \\
\hline $\boldsymbol{\theta}$ & 47.88344 & $(1.133654,2.786414)$ \\
\hline $\mathbf{u}(\mathbf{t})$ & 1.777310 & $(0.003788341,4.777899)$ \\
\hline $\mathbf{m}(\mathbf{t})$ & 0.1345374 & $(0.2092970,263.9678)$ \\
\hline $\mathbf{u}_{\mathbf{c}}(\mathbf{t})$ & 7.432878 & $(8.04828334,0.1186759)$ \\
\hline $\mathbf{m}_{\mathbf{c}}(\mathbf{t})$ & 0.07569721 & $(12.11912,29.78765)$ \\
\hline $\mathbf{N}(\mathbf{t})$ & 13.21053 & $(1.091529 \mathrm{e}-12,2.875905 \mathrm{e}-05)$ \\
\hline $\mathbf{R}(\mathbf{t}=\mathbf{2 5 1})$ & 19 & $(0.004326917,0.00801076)$ \\
\hline $\mathbf{t}(\mathbf{R}=\mathbf{0 . 9})$ & $5.602796 \mathrm{e}-09$ & \\
\hline
\end{tabular}


Table 15 Point and Interval estimates of reliability indices using LLP

\begin{tabular}{|c|c|c|}
\hline & Point Estimate & Interval Estimate \\
\hline $\boldsymbol{a}$ & -4.052577 & $(-4.868371,-3.373485)$ \\
\hline $\boldsymbol{b}$ & 0.0079844 & $(0.004332011,0.01471618)$ \\
\hline $\mathbf{u}(\mathbf{t})$ & 0.1289291 & $(0.05928362,0.2803932)$ \\
\hline $\mathbf{m}(\mathbf{t})$ & 7.756198 & $(0.03384319,0.0915479)$ \\
\hline $\mathbf{u}_{\mathbf{c}}(\mathbf{t})$ & 0.05566213 & $(10.92324,29.54805)$ \\
\hline $\mathbf{m}_{\mathbf{c}}(\mathbf{t})$ & 17.96554 & $(8.49464,22.97852)$ \\
\hline $\mathbf{N}(\mathbf{t})$ & 13.97119 & $\left(8.191668 \times 10^{-10}, 0.0008941341\right)$ \\
\hline $\mathbf{R}(\mathbf{t}=\mathbf{2 5 1})$ & $8.5583 \times 10^{-7}$ & $(2.832404,12.37691)$ \\
\hline $\mathbf{t}(\mathbf{R}=\mathbf{0 . 9})$ & 5.920844 & \\
\hline
\end{tabular}

Table 16 Comparison on the basis of AIC and BIC

\begin{tabular}{|c|c|c|l|}
\hline & AIC & BIC & \multicolumn{1}{c|}{ Conclusion } \\
\hline PLP & 134.8441 & 136.7329 & $\begin{array}{l}\text { PLP is better than LLP for } \\
\text { this data. }\end{array}$ \\
\hline LLP & 135.8941 & 137.783 & \\
\hline
\end{tabular}

\section{E: 1500 points UP $(n=21)$ :}

Cumulative number of months:

$13,15,36,102,109,152,156,167,176,182,184,188,191,198,200,201,219,220,224$,

$231,236$.

Convexity of the curve in Fig. 13 means presence of trend in the data. The TTT plot in Fig. 14 indicates that the data initially shows increasing failure rate, then there is an improvement in the system and then after again the failure rate is increasing. That means the trend is present in the data. Nonlinearity of Duane plot in Fig. 15 indicates that the process is not PLP.

From Table 17, it can be concluded that the data can be modelled using an NHPP. Table 18 and 19 consists of point and interval estimates of model parameters and reliability indices for PLP and LLP respectively. Table 20 shows that LLP is a more suitable model for describing the 1500 points up data.
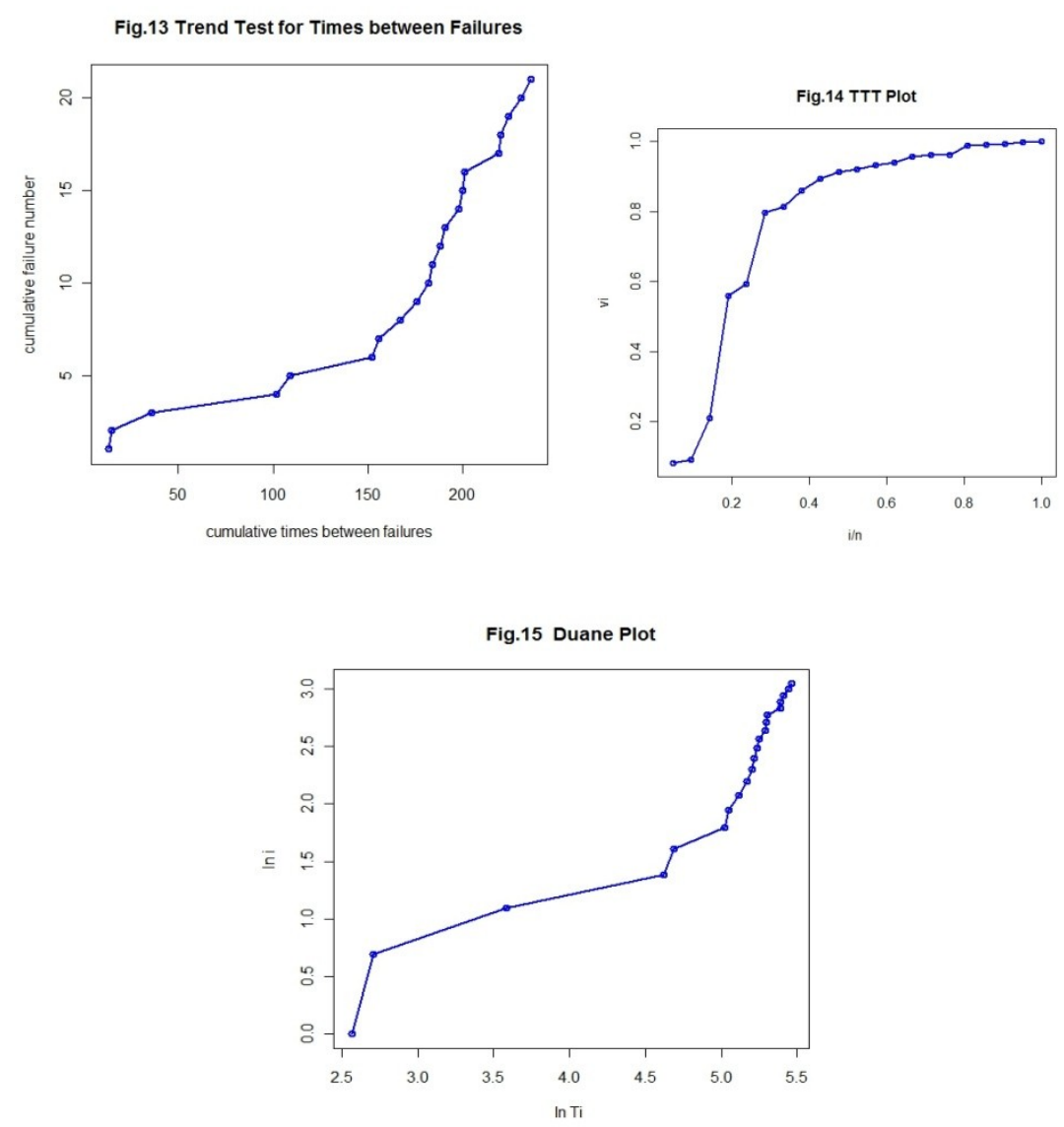
Table 17 Goodness of fit Tests

\begin{tabular}{|l|c|c|c|}
\hline & Test statistic value & Critical Value & Conclusion \\
\hline MIL-HDBK-189 Test & 24.61454 & 55.75848 & Process is NHPP \\
\hline CVM Test & 0.2232724 & 0.217 & Process is not PLP \\
\hline Laplace Test & 2.63888 & 1.96 & Process is NHPP \\
\hline Lewis-Robinson Trend Test & 1.928144 & 1.96 & Process is NHPP \\
\hline Standardized Q & 2.652819 & -1.23 & Process is NHPP \\
\hline
\end{tabular}

Table 18 Point and Interval estimates of reliability indices using PLP

\begin{tabular}{|c|c|c|}
\hline $\boldsymbol{\theta}$ & Point Estimate & Interval Estimate \\
\hline $\boldsymbol{\beta}$ & 39.62884 & $(17.74845,88.48349)$ \\
\hline $\mathbf{u}(\mathbf{t})$ & 1.706308 & $(1.112517,2.617028)$ \\
\hline $\mathbf{m}(\mathbf{t})$ & 0.1518325 & $(0.2844166,152.5160)$ \\
\hline $\mathbf{u}_{\mathbf{c}}(\mathbf{t})$ & 6.586205 & $(0.05801716,0.1364766)$ \\
\hline $\mathbf{m}_{\mathbf{c}}(\mathbf{t})$ & 0.08898305 & $(7.327265,17.23628)$ \\
\hline $\mathbf{N}(\mathbf{t})$ & 11.23810 & $(13.69205,32.20847)$ \\
\hline $\mathbf{R}(\mathbf{t}=\mathbf{2 3 6})$ & 21 & $(9.52903 \mathrm{e}-14,6.033691 \mathrm{e}-06)$ \\
\hline $\mathbf{t}(\mathbf{R}=\mathbf{0 . 9})$ & $7.58256 \mathrm{e}-10$ & $(0.004900099,0.009294637)$ \\
\hline
\end{tabular}

Table 19 Point and Interval estimates of reliability indices using LLP

\begin{tabular}{|c|c|c|}
\hline & Point Estimate & Interval Estimate \\
\hline $\boldsymbol{a}$ & -3.790502 & $(-6.858874,-2.094791)$ \\
\hline $\boldsymbol{b}$ & 0.01036189 & $(0.003111149,0.03451097)$ \\
\hline $\mathbf{u}(\mathbf{t})$ & 0.2605148 & $(1.639597,8.98666)$ \\
\hline $\mathbf{m}(\mathbf{t})$ & 3.838554 & $(0.06096711,0.1552754)$ \\
\hline $\mathbf{u}_{\mathbf{c}}(\mathbf{t})$ & 0.09729692 & $(6.440171,16.40229)$ \\
\hline $\mathbf{m}_{\mathbf{c}}(\mathbf{t})$ & 10.27782 & $(14.38824,36.64499)$ \\
\hline $\mathbf{N}(\mathbf{t})$ & 22.96207 & $\left(2.324428 \times 10^{-15}, 4.88741 \times 10^{-6}\right)$ \\
\hline $\mathbf{R}(\mathbf{t}=\mathbf{2 3 6})$ & $1.065854 \times 10^{-10}$ & $(0.4932605,42.08084)$ \\
\hline $\mathbf{t}(\mathbf{R}=\mathbf{0 . 9})$ & 4.555965 & \\
\hline
\end{tabular}

Table 20 Comparison on the basis of AIC and BIC

\begin{tabular}{|c|c|c|c|}
\hline & AIC & BIC & Conclusion \\
\hline PLP & 142.5545 & 144.6435 & LLP is better than PLP for \\
this data.
\end{tabular}

F: 1500 points DOWN $(n=10)$ :

Cumulative number of months:

18, 115, 185, 205, 206, 210, 213, 214, 217, 248.
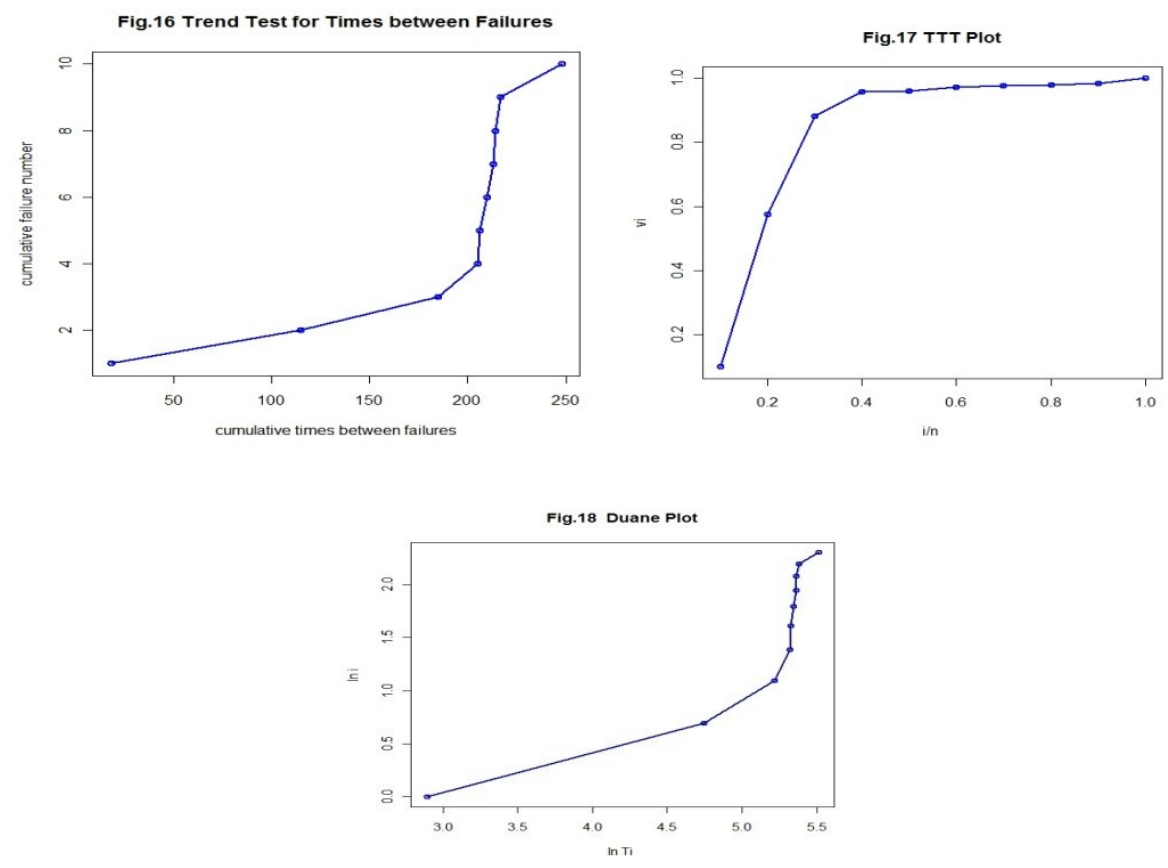
Convexity of the curve in Fig. 16 means presence of trend in the data. The TTT plot in Fig. 17 indicates that the data initially shows increasing failure rate, then there is an improvement in the system which means the trend is present in the data. Linearity of Duane plot indicates PLP, so here from Fig. 18, we can say that the process is not PLP.

From Table 21, it can be concluded that the data can be modelled using an NHPP. Table 22 and 23 consists of point and interval estimates of model parameters and reliability indices for PLP and LLP respectively. Table 24 shows that LLP is a more suitable model for describing the 1500 points up data.

Table 21 Goodness of fit Tests

\begin{tabular}{|l|c|c|c|}
\hline & Test statistic value & Critical Value & Conclusion \\
\hline MIL-HDBK-189 Test & 9.32008 & 28.8693 & Process is NHPP \\
\hline CVM Test & 0.2395520 & 0.212 & Process is not PLP \\
\hline Laplace Test & 2.174376 & 1.96 & Process is NHPP \\
\hline Lewis-Robinson Trend Test & 1.715602 & 1.96 & Process is NHPP \\
\hline Standardized Q & 1.8047378 & -1.1664 & Process is NHPP \\
\hline
\end{tabular}

Table 22 Point and Interval estimates of reliability indices using PLP

\begin{tabular}{|c|c|c|}
\hline & Point Estimate & Interval Estimate \\
\hline $\boldsymbol{\theta}$ & 84.81012 & $(41.07265,175.1228)$ \\
\hline $\mathbf{\beta}$ & 2.145904 & $(1.154601,3.98831)$ \\
\hline $\mathbf{u}(\mathbf{t})$ & 0.0865284 & $(0.01644317,8122.638)$ \\
\hline $\mathbf{u}_{\mathbf{c}}(\mathbf{t})$ & 11.55690 & $(0.02169551,0.07494227)$ \\
\hline $\mathbf{m}_{\mathbf{c}}(\mathbf{t})$ & 0.04032258 & $(13.34360,46.09249)$ \\
\hline $\mathbf{N}(\mathbf{t})$ & 24.8 & $(5.380486,18.58568)$ \\
\hline $\mathbf{R}(\mathbf{t}=\mathbf{2 4 8})$ & 10 & $(9.231454 \mathrm{e}-08,0.02232751)$ \\
\hline $\mathbf{t}(\mathbf{R}=\mathbf{0 . 9})$ & $4.539993 \mathrm{e}-05$ & $(0.003093906,0.005517286)$ \\
\hline
\end{tabular}

Table 23 Point and Interval estimates of reliability indices using LLP

\begin{tabular}{|c|c|c|}
\hline & Point Estimate & Interval Estimate \\
\hline $\boldsymbol{a}$ & -4.662222 & $(-10.75455,-2.021127)$ \\
\hline $\boldsymbol{b}$ & 0.01354306 & $(0.001455788,0.073753)$ \\
\hline $\mathbf{u}(\mathbf{t})$ & 0.1233816 & $(0.03207657,0.4745839)$ \\
\hline $\mathbf{m}(\mathbf{t})$ & 8.104935 & $(0.02177636,0.09027276)$ \\
\hline $\mathbf{u}_{\mathbf{c}}(\mathbf{t})$ & 0.04433748 & $(11.07754,45.92137)$ \\
\hline $\mathbf{m}_{\mathbf{c}}(\mathbf{t})$ & 22.55428 & $(5.400536,22.38764)$ \\
\hline $\mathbf{N}(\mathbf{t})$ & 10.99569 & $\left(6.750209 \times 10^{-9}, 0.04168161\right)$ \\
\hline $\mathbf{R}(\mathbf{t}=\mathbf{2 4 8})$ & $1.677378 \times 10^{-5}$ & $(0.2376687,468.817)$ \\
\hline $\mathbf{t}(\mathbf{R}=\mathbf{0 . 9})$ & 10.55572 & \\
\hline
\end{tabular}

Table 24 Comparison on the basis of AIC and BIC

\begin{tabular}{|c|c|c|c|}
\hline & AIC & BIC & Conclusion \\
\hline PLP & 83.62557 & 84.23074 & LLP is better than PLP for \\
this data.
\end{tabular}

\section{Conclusions}

From the trend analysis, it is clear that the assumption of i.i.d. is, in general, not valid for the TBFs of the stock prices. The assumption that the NHPP adequately describes TBFs of stock prices is validated both graphically and analytically. The values of shape parameter $\beta$ of PLP is greater than 1 for all datasets, which clearly indicates that the failure intensities are increasing, and their maintenance policies should be changed or further reinforcement should be made. In this study, using both models, PLP and LLP, the point MLE and interval estimates for the parameters and the reliability indices are computed based on asymptotic results. It can be seen that for the sensex data regarding 500 points up, 500 points down, 1000 points up, 1500 points up and 1500 points down, LLP may be a suitable model; whereas for the sensex data regarding 1000 points down, PLP may be a suitable model.

\section{References:}

[1] Mishra, P. K., Das K. B. and Pradhan, B. B., Empirical evidence on Indian stock market efficiency in context of the global financial crisis. Global Jr. of Finance and Management, 1(2), 2009, 149-157.

[2] Mishra, P. K., Indian capital market - Revisiting market efficiency. Indian Jr. of Capital Markets, 2(5), 2009.

[3] Zhou, H. and Rigdon. S. E., Duration dependence in U. S. business cycles: An analysis using the modulated power law process. $J r$. of Economics and Finance, 32(1), 2008, 25-34.

[4] Zhou, H. and Rigdon. S. E., Duration dependence in bull and bear stock markets. Modern Economy, 2, 2011, 279-286.

[5] Ascher, H. E. and Feingold, H.,Repairable Systems Reliability - Modeling, Inference, Misconceptions and Their Cause (Marcel Dekker, New York, 1984). 
[6] Calabria, R. and Pulcini, G., Inference and test in modeling the failure/repair process of repairable mechanical equipments. Reliab. Eng. Syst. Saf., 67, 2000, 41-53.

[7] Lawless, J. F.,Statistical Models and Methods for Lifetime Data Analysis (John Wiley, New York, 1982).

[8] Muralidharan, K., A review of repairable systems and point process models. ProbStat Forum, 1, 2008, 26-49.

[9] Lawless, J. F. and Thiagarajah, K., A point-process model incorporating renewals and time trends, with application to repairable systems. Technometrics, 38(2), 1996, 131-138.

[10] Cox, D. R., Some statistical methods connected with series of events. Jr. of Royal Statistical Soc. B, 17, 1955, 129-164.

[11] Cox, D. R., The statistical analysis of dependencies in point processes. In Stochastic Point Processes, Ed. P. A. W. Lewis, 1972, 55-66. New York: Wiley.

[12] Cox, D. R. and Lewis, P. A., The statistical analysis of series of events (Metheun, London, 1966).

[13] Rigdon, S. E. and Basu, A. P., The power law process: A model for the reliability of repairable systems. Jr. of Quality Technology, 20, 1989, 251-260.

[14] Crow, L. H., Reliability analysis for complex repairable systems. In Reliabilityand Biometry. F. Proschan and R. J. Serfling, Editors. Society for Industrial and Applied Mathematics (SIAM), Philadelphia, 1974, 379-410.

[15] Crow, L. H., Confidence interval procedures for the Weibull process with applications to reliability growth. Technometrics, 24,1982, 67-72.

[16] Baker, R. D., Some new tests of the power law process. Technometrics, 38 (3), 1996, 256-265.

[17] Rigdon, S. E. and Basu, A. P., Estimating the intensity function of a power law process at the current time in time truncated case. Commun. Statist.-Simulation, 19, 1990, 1079-1104.

[18] Wang, Z. M. and Xu X., Log-linear process modeling for repairable systems with time trends and its applications in reliability assessment of NC machine tools: Case Study. Jr. of Risk and Reliability, 227 (1), 2013, 55-65.

[19] Muralidharan, K., Shah, R., Conditional test for trend in Cox process. IAPQR Trans., 36 (1), 2011, 95-109.

[20] Nelson, W., An application of graphical analysis of repair data. Qual. Reliab. Eng. Int., 14(1), 1998, 49-52.

[21] Duane, J. T., Learning curve approach to reliability monitoring. IEEE Trans., A-2, 1964, 563-566.

[22] Klefsjo, B. and Kumar, U., Goodness-of-fit tests for the power-law process based on the TTT-plot. IEEE trans.on Reliability, R-41 (4), 1992, 593-598.

[23] MIL-HDBK-189, Reliability growth management (Headquarters, US Army Communication Research and Development Command, ATTN: DRDCO-PT, Fort Monmouth, NJ, 1981).

[24] Jani, P. N., Shanubhogue, A. and Muralidharan, K., Some tests for a Poisson process and their powers with respect to trend in the intensity. Commun. Statist.- Theory and Methods, 26 (11), 1997, 2671-2688. 